\title{
Excited Eigenstates and Strength Functions for Isolated Systems of Interacting Particles
}

\author{
V.V.Flambaum ${ }^{1}$ and F.M.Izrailev ${ }^{2}$ \\ ${ }^{1}$ School of Physics, University of New South Wales, Sydney 2052, Australia \\ ${ }^{2}$ Instituto de Física, Universidad Autonoma de Puebla, Apdo. Postal J-48, Puebla, 72570 México
}

(July 14, 2018)

\begin{abstract}
Eigenstates in finite systems such as nuclei, atoms, atomic clusters and quantum dots with few excited particles are chaotic superpositions of shell model basis states. We study criterion for the equilibrium distribution of basis components (ergodicity, or Quantum Chaos), effects of level density variation and transition from the Breit-Wigner to the Gaussian shape of eigenstates and strength functions. In the model of $n$ interacting particles distributed over $m$ orbitals, the shape is given by the Breit-Wigner function with the width in the form of gaussian dependence on energy.
\end{abstract}

PACS numbers: 05.45.+b, 31.25.-v, 31.50.+w, 32.30.-r

Introduction. Recently, based on chaotic structure of eigenstates, statistical approach has been developed in [1] which allows to find distribution of occupation numbers for single-particles states, expectation values of different operators, transition amplitudes between chaotic states and degree of an enhancement of weak interactions. The main quantity in this approach is the form of the distribution of shell model basis components in chaotic eigenstates. In recent numerical studies of the Ce atom [2], the $s-d$ nuclear shell model [5] and random two-body interaction model [3.6] it was found that typical shape of exact eigenstates practically does not depend on a particular many-body system and has a universal form which essentially depends on few parameters.

In this paper, we develop a method for the description of the form of chaotic eigenstates and strength functions without diagonalization of huge many-body Hamiltonian matrices.

We follow the standard way according to which the total Hamiltonian for $n$ Fermi-particles distributed over $m$ single-particles states is written as a sum of two terms,

$$
H=H_{0}+V=\sum_{s=1}^{m} \epsilon_{s} a_{s}^{+} a_{s}+\frac{1}{2} \sum V_{p q r s} a_{p}^{+} a_{q}^{+} a_{r} a_{s}
$$

Here the "unperturbed" Hamiltonian $H_{0}$ incorporates an effect of the mean field, $\epsilon_{s}$ are the energies of singleparticle states calculated in this field, $a_{s}^{+}, a_{s}$ are creationannihilation operators, and $V$ stands for the residual twobody interaction (the difference between the exact and mean field Hamiltonians).

Exact ("compound") eigenstates $|i\rangle$ of the Hamiltonian $H$ can be expressed in terms of simple shell-model basis states $|k\rangle$ (eigenstates of $H_{0}$ ):

$$
|i\rangle=\sum_{k} C_{k}^{(i)}|k\rangle ; \quad|k\rangle=a_{k_{1}}^{+} \ldots a_{k_{n}}^{+}|0\rangle
$$

These compound eigenstates $|i\rangle$ are formed by the residual interaction $V$; in complex systems they typically contain large number $N_{p c} \gg 1$ of the so-called principal components $C_{k}^{(i)}$ which fluctuate "randomly" as a function of indices $i$ and $k$. The shape of exact eigenstates is given by the "spreading function" $F$ (in what follows, the $F$ - function ),

$$
F_{k}^{(i)} \equiv \overline{\left|C_{k}^{(i)}\right|^{2}} \simeq F\left(E_{k}, E^{(i)}\right)
$$

where $E_{k}$ is the unperturbed energy and $E^{(i)}$ is the perturbed one.

Equations for strength function and spreading width. For a weak interaction between particles the shape of chaotic eigenstates is known to be well described by the Breit-Wigner form. However, with an increase of the interaction strength, the average shape of the eigenstates ( $F$-function) changes from the Breit-Wigner one to that close to the Gaussian with the exponential tails 2. 4 . In order to reduce the distortion effect due to nonconstant density of states, in what follows we consider the so-called "strength function" $P_{k}(E)$ which is also known in literature as "local spectral density of states" (LDOS),

$$
P_{k}(E) \equiv F\left(E_{k}, E\right) \rho(E)
$$

Note that the $F$-function gives the shapes of both exact eigenstates and the LDOS, depending on what is fixed, the total energy $E^{(i)}$ or the unperturbed one, $E_{k}$.

The equations for $P_{k}(E)$ can be obtained on the base of the method presented in [7,8]. Let us choose some basis component $|k\rangle$ and diagonalize the Hamiltonian matrix without this component. Thus, the problem is reduced to the interaction of this component with the exact eigenstates $|i\rangle$ described by the matrix elements $V_{k i}$.

The solution can be written by making use of the average over a small energy interval $\Delta$ (see details in [7]),

$$
P_{k}(E)=\frac{1}{2 \pi} \frac{\Gamma_{k}(E)}{\left(E_{k}+\delta_{k}-E\right)^{2}+\left(\Gamma_{k}(E) / 2\right)^{2}}
$$




$$
\begin{gathered}
\Gamma_{k}(E) \simeq 2 \pi \overline{\left|V_{k i}\right|^{2}} \rho(E) \\
\delta_{k}=\sum_{i} \frac{\left|V_{k i}\right|^{2}\left(E-E^{(i)}\right)}{\left(E-E^{(i)}\right)^{2}+(\Delta / 2)^{2}}
\end{gathered}
$$

It is easy to recognize in the energy shift $\delta_{k}$ the modified second order correction to the unperturbed energy level. For the calculation of the shape of the eigenvector $\mid i>$ one should substitute the exact energy $E=E^{(i)}=$ $E_{i}+\delta_{i}$. The difference $\delta_{i}-\delta_{k}$ is negligible if the interaction is not very strong.

One should stress that the summation in the above equations are performed over exact states. We would like to express the result in terms of the basis states only, this allows us to solve the problem without diagonalization of the Hamiltonian matrix $H_{k p}$. To do this, we express exact eigenstates $\mid i>$ in terms of the basis components,

$$
\left|V_{k i}\right|^{2}=\sum_{p}\left|C_{p}^{(i)}\right|^{2}\left|H_{k p}\right|^{2}+\sum_{p \neq q} C_{q}^{(i) *} C_{p}^{(i)} H_{k p} H_{q k}
$$

with $H_{k p}$ standing for non-diagonal Hamiltonian matrix elements. If coefficients $C_{p, q}^{(i)}$ can be treated as random variables, the second term vanishes after averaging. Substitution of Eq. (8) into Eqs.(6, 7) gives

$$
\begin{gathered}
\Gamma_{k}(E)=2 \pi \sum_{p \neq k}\left|H_{k p}\right|^{2} P_{p}(E)= \\
\sum_{p \neq k}\left|H_{k p}\right|^{2} \frac{\Gamma_{p}(E)}{\left(E_{p}+\delta_{p}-E\right)^{2}+\left(\Gamma_{p}(E) / 2\right)^{2}} \\
\delta_{k}=\sum_{p \neq k}\left|H_{k p}\right|^{2} \int d E^{(i)} \frac{P_{p}\left(E^{(i)}\right)}{E-E^{(i)}} \simeq \\
\sum_{p \neq k} \frac{\left|H_{k p}\right|^{2}\left(E-E_{p}-\delta_{p}\right)}{\left(E-E_{p}-\delta_{p}\right)^{2}+\left(\Gamma_{p}(E) / 2\right)^{2}}
\end{gathered}
$$

where the integral is taken as the principal value. Last equality is valid in the approximation of slow variation of $\Gamma_{p}(E)$ and $\delta_{p}$. The equations for $\Gamma_{k}(E)$ and $\delta_{k}$ allow to calculate the strength function (5) from the unperturbed energy spectrum and matrix elements of the total Hamiltonian $H$.

Criterion of statistical equilibrium. Let us discuss the conditions under which self-consistent solution of the equations $(5,9,10)$ exists. There are four important parameters in this problem: the spreading width of a basis component $\Gamma$, the effective band width $\sigma$ of the Hamiltonian matrix $H_{p q}$, the interval between the many - body energy levels $D=\rho^{-1}$ and the interval $d_{f}=\rho_{f}^{-1}$ between the final basis states $\mid p>$ which can be connected with a particular basis component $\mid k>$ by the two-body interaction. The ratio $D / d_{f}$ is exponentially small [3] since all the basis states $\mid p>$ which differ from $\mid k>$ by position of more than two particles, have zero matrix elements $H_{k p}$ and do not contribute to the Eqs. (9, 10).

First, let us consider Eqs. (9, 10) for a strong enough interaction, $\Gamma>d_{f}$. In this case the number of effectively large terms in the sums is large, $N_{f} \sim \Gamma / d_{f}$, and fluctuations of $\Gamma$ are small, $\delta \Gamma \sim \Gamma / \sqrt{N_{f}}$. Therefore, Eq. (9) can be written as

$$
\Gamma_{k}(E) \simeq 2 \pi \overline{\left|H_{k p}\right|^{2}} \rho_{f}(\tilde{E})
$$

where $\tilde{E}=E-\delta$. The energy shift $\delta \equiv<\delta_{p}>$ can be neglected in the case of $\Gamma<<\sigma$. In order to perform the summation over $p$, we assumed that $\Gamma(E)$ and $\rho_{f}(E)$ vary slowly within the energy interval of the size $\Gamma$. Thus, in order to have large number of final states $N_{f} \sim 2 \pi H_{k p}^{2} / d_{f}^{2}$ and statistical equilibrium (small fluctuations of $\Gamma$ ), one needs $H_{k p}>>d_{f}$. In this case chaotic components of exact eigenfunctions in the unperturbed many-particle basis ergodically fill the whole energy shell of the width $\Gamma$, with Gaussian fluctuations of the coefficients $C_{k}^{(i)}$ with the variance given by the $F$-function (3) (see also [7,2]).

With the decrease of the ratio $H_{k p} / d_{f}$ the fluctuations of $\Gamma$ increase and for $H_{k p}<d_{f}$ the smooth selfconsistent solution of Eqs.(9) disappears. Indeed, in this case $\Gamma_{p}$ in the denominator can be neglected and the sum in (9) is dominated by one term with a minimal energy $E-E_{p} \sim d_{f}$. Therefore, for a typical basis state $\mid k>$ formally one gets $\Gamma_{k} \sim \Gamma_{p}\left(H_{k p} / d_{f}\right)^{2}<<\Gamma_{p}$. This contradicts to the equilibrium condition according to which all components are "equal" $\left(\Gamma_{k} \sim \Gamma_{p}\right)$.

One should stress that the absence of a smooth solution for the shape of the eigenstates and the strength function does not mean that the number of principal components in exact eigenstates is small. However, the distribution of the components is not ergodic: there are many "holes" inside exact eigenstates which occupy the energy shell of the width $2 \pi \overline{\left|H_{k p}\right|^{2}} \rho_{f}(E)$ (see [10,4). In such a situation, very large (non-Gaussian) fluctuations of $C_{k}^{(i)}$ are typical.

It is important that ensemble averaging in this problem is not equivalent to the energy average for a specific Hamiltonian matrix. For example, the average over the single-particle spectrum leads to variation of energy denominators in (9) and can fill the holes in the $F$-function even for $\Gamma<d_{f}$.

Transition from the Breit-Wigner type to the Gaussian-like strength function. In principal, the set of equations (9, 10) for the shape of the strength function $P_{k}(E)$ defined by Eq.(5), can be solved numerically having the unperturbed many-body spectrum and matrix elements $H_{k p}$ of the total Hamiltonian. However, for relatively large number of particles (practically, for 
$n \geq 4$ ), one can find an approximate analytical solution of the problem.

First, we note that the spreading width $\Gamma(E)$ in the expression (5) for the strength function can be a strong function of excitation energy $E$ due to the variation of the density of the final states $\left.\rho_{f}(E)\right)=\left(d_{f}\right)^{-1}$ in Eq. (11). It is well-known that at small $E$ the basis component with one excited particle has $\Gamma(E) \propto E^{2} / d_{0}$ where $d_{0}$ is the interval between single-particle energy levels. For typical case of $n^{\star} \sim\left(E / d_{0}\right)^{1 / 2}$ excited particles the spreading width can be estimated as $\Gamma(E) \propto\left(d_{f}\right)^{-1} \sim$ $\left(d_{0}\right)^{-1}\left(E / d_{0}\right)^{3 / 2}[9]$. Below we show that at higher energies far from the ground state, the energy dependence of $\rho_{f}(E)$ and $\Gamma(E)$ can be quite close to the Gaussian. Note that the Gaussian form typically occurs in "statistical spectroscopy" 11] when neglecting the mean field term in Eq.(1).

In the model (11) the density $d_{f}$ is defined by transitions between those basis states which differ by the position of one or two particles only, therefore, $\rho_{f}(E)=$ $\rho_{f}^{(1)}(E)+\rho_{f}^{(2)}(E)$.

Let us estimate the density $\rho_{f}^{(2)}$ determined by the energy difference $\omega_{p k}^{(2)}$ between the states $\mid p>$ and $\mid k>$ which differ by the position of two particles,

$$
\begin{gathered}
\omega_{p k}^{(2)}=\epsilon_{\alpha}^{(p)}+\epsilon_{\beta}^{(p)}-\epsilon_{\gamma}^{(k)}-\epsilon_{\delta}^{(k)} \\
+\sum_{\nu \neq \alpha, \beta, \gamma, \delta}\left(V_{\alpha \nu}+V_{\beta \nu}-V_{\gamma \nu}-V_{\delta \nu}\right)+V_{\alpha \beta}-V_{\gamma \delta}
\end{gathered}
$$

Here the summation is taken over $n-2$ occupied orbitals and $V_{\alpha \nu}$ are the diagonal matrix elements of the residual interaction between the particles located at the orbitals $\alpha$ and $\nu$. The matrix elements of residual interaction are assumed to be random with the zero mean.

For large number of fluctuating terms in the Eq.(12) the distribution of $\omega$ is close to the gaussian form. Strictly speaking, this is correct if the contribution of $4 n-6$ interaction terms to the frequency Eq.(12) is strong. However, even four single-particle energy terms give the distribution which is close to the Gaussian. The same conclusion is reasonable also for single-particle transition density $\rho_{f}^{(1)}$, thus, the general expression reads as

$$
\rho_{f}^{(1,2)}(\tilde{E}) \simeq K\left(2 \pi \sigma^{2}\right)^{-1 / 2} \exp \left(-\frac{\left(\tilde{E}-E_{k}-\bar{\omega}\right)^{2}}{2 \sigma^{2}}\right) .
$$

The normalization parameter $K$ stands for the number of one or two-particle transitions, $K=K_{1}=n(m-n)$ and $K=K_{2}=n(n-1)(m-n)(m-n-1) / 4$ correspondingly [3].

From Eq.(12) the estimate for the average frequency of two-particle transitions reads as $\overline{\omega^{(2)}}=2\left(\overline{\epsilon_{p}}-\overline{\epsilon_{k}}\right) \approx$ $2 m /(m-n)\left(\bar{\epsilon}-E_{k} / n\right)$ where $\overline{\epsilon_{k}}=E_{k} / n$ is the average single-particle energy in the basis state $\mid k>$ containing $n$ particles, $\bar{\epsilon}$ is the single-particle energy averaged over all $m$ orbitals. Average energy of the empty orbitals $\overline{\epsilon_{p}}$ can be found from the relation $m \bar{\epsilon}=\overline{\epsilon_{k}} n+\overline{\epsilon_{p}}(m-n)$.

The variance of $\rho_{f}^{(2)}(E)$ for two-particle transitions is equal to $\sigma_{2}^{2}=2 \sigma_{p}^{2}+2 \sigma_{k}^{2}+(4 n-6) V^{2} \approx 2 \sigma_{\epsilon}^{2}+(4 n-6) V^{2}$ where $\sigma_{\epsilon}^{2}$ is the variance of single-particle spectrum, and $V^{2}$ is the variance of non-diagonal matrix elements of the two-body residual interaction. Note that in the case of $n<<m$ for low-lying states the variance of the occupied orbital energies $\sigma_{k}^{2}$ is small and the variance of empty orbital energies is $\sigma_{p}^{2} \sim \sigma_{\epsilon}^{2}$.

Similar, the density $\rho_{f}^{(1)}$ is also approximated by the Eq.(13), with $K=K_{1}, \overline{\omega^{(1)}} \approx m /(m-n)\left(\bar{\epsilon}-E_{k} / n\right)$ and $\sigma_{1}^{2}=\sigma_{p}^{2}+\sigma_{k}^{2}+2(n-1) V^{2} \approx \sigma_{\epsilon}^{2}+2(n-1) V^{2}$.

Thus, the width $\Gamma(E)$ is given by the following expression, $\Gamma=2 \pi\left[(n-1) V^{2} \rho_{f}^{(1)}+V^{2} \rho_{f}^{(2)}\right]$. Since for singleparticle transitions the summation in $H_{k p}=\sum_{\nu} V_{\alpha \nu \rightarrow \gamma \nu}$ is performed over occupied orbitals, the factor $n-1$ appears in the above relation. Typically, the ratio $K_{2} /\left[(n-1) K_{1}\right]=(m-n-1) / 4$ is larger than 1 , therefore, the two-particle transitions dominate. In this case we can neglect the differences in $\bar{\omega}$ and $\sigma$ for two-particle and one-particle transitions and combine two terms into one. As a result, the spreading width is described by the simple Gaussian formula

$$
\Gamma_{k}(E) \simeq 2 \pi(\Delta E)_{k}^{2} \frac{1}{\sqrt{2 \pi \sigma_{k}^{2}}} \exp \left\{-\frac{\left(\tilde{E}-E_{k}-\overline{\omega_{k}}\right)^{2}}{2 \sigma_{k}^{2}}\right\}
$$

where $\tilde{E}=E-\delta$. Here $(\Delta E)_{k}^{2}$ is the variance of the strength function which can be defined through its average value [3:4],

$$
\overline{(\Delta E)_{k}^{2}}=\overline{\sum_{p \neq k} H_{k p}^{2}}=V^{2} n(n-1)(m-n)(3+m-n) / 4
$$

and $\omega_{k}$ and $\sigma_{k}$ are close to that for the two-particle transitions. The maximum of $\rho_{f}(E)$ and $\Gamma(E)$ is shifted by $\left|\overline{\omega_{k}}\right|$ towards the center of the spectrum compared to the maximum of Breit-Wigner function. This leads to some distortion of the strength function Eq.([5) and the shape of the eigenstates, which is especially large at the bottom of the spectrum.

Thus, we have demonstrated that if the interaction is small $\left(\Gamma \ll \sigma_{k}\right)$, the strength function has the BreitWigner shape with the broad gaussian envelope originating from $\Gamma_{k}(E)$ in the numerator of Eq. (5). It is easy to check that this envelope is, indeed, needed in order to provide the correct value $(\Delta E)_{k}^{2}$ for the second moment of the strength function (note, that the Breit-Wigner shape has infinite second moment which is unphysical).

When the interaction $V$ increases one needs to take into account one more contribution to the broadening of the shape of $\Gamma(E)$. It is given by the width of the 
strength function $P_{p}(E)$ in Eq.(9) (it was neglected in Eq.(11)). Taking into account this width we can give an estimate $\sigma_{k}^{2} \simeq \sigma_{2}^{2}+\overline{\Gamma_{p}^{2}}$. With further increase of interaction, where the shape of $P_{p}(E)$ is close to the Gaussian, we have $\sigma_{k}^{2} \simeq \sigma_{2}^{2}+(\Delta E)_{k}^{2}$.

Direct numerical study of the model (11) with $n=6$ Fermi-particles and $m=13$ orbitals shows that the above analytical expressions give quite a good description of the shape of the strength function $P_{k}(E)$ as well as of the energy dependence of the spreading width. The unperturbed single-particle spectrum has been chosen at random, with $d_{0}=1$ and $\epsilon_{s} \approx d_{0} s$. The size of the Hamiltonian matrix is $N=C_{m}^{n}=1716$ and we specify the unperturbed state $\mid i_{0}>$ with $i_{0}=280$.

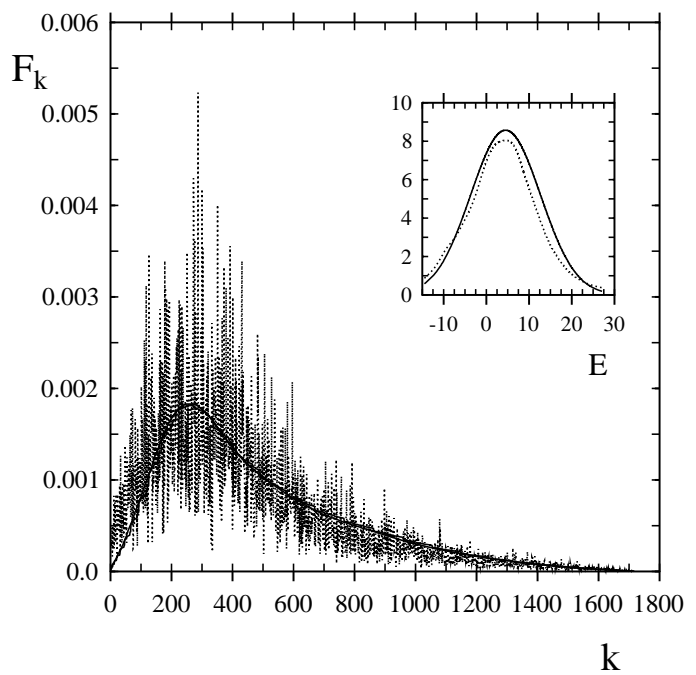

FIG. 1. The $F$-function (3) in the basis representation. Broken line is the result of numerical diagonalization of the Hamiltonian matrix; to reduce the fluctuations, we take the average over 10 matrices $H_{i k}$ with different two-body random interaction with $V^{2} \approx 0.15$. Two smooth curves correspond to the computation of Eq. (4) with $\Gamma_{k}(E)$ given by Eqs. (9.10) and by Eq.(14); they practically coincide. The inset shows the dependence $\Gamma_{k}(E)$ itself; full curve is the expression (14), the dashed curve is the computation from Eqs. $(9,10)$.

In the estimates above we assumed that $\Gamma<<\sigma$ where $\sigma$ is the effective energy band width of the Hamiltonian matrix, see Eq. (14). When $\Gamma \sim \sigma$, the (Gaussian) variation of $\Gamma(E)$ in the numerator of the strength function in Eq.(5) becomes as important as the variation of the BreitWigner energy denominator $\left(E-E_{k}\right)^{2}+(\Gamma / 2)^{2}$. At this point, $\Gamma \approx \sigma$, the transition from the Breit-Wigner type to Gaussian-type shape of the eigenstates takes place. We still can use Eqs.(9, 10), and (4) in order to calculate (numerically) $\Gamma(E), P_{k}(E)$ and $F\left(E, E_{k}\right)$, using $\Gamma$ from Eq.(14) with $\sigma_{k}^{2} \simeq \sigma_{2}^{2}+(\Delta E)_{k}^{2}$ as the zero approximation in the right-hand side of Eqs.(9, 10).

Acknowledgements. VVF is grateful to ICTP (Triest), Princeton University and NEC Research Institute for hospitality. VVF acknowledges the support from Australian Research Council, and FMI acknowledges the support from the CONACyT Grant No. 26163-E (Mexico). Both authors are very thankful to Prof. V.Zelevinsky for valuable comments and kind hospitality during the stay in MSU Cyclotron laboratory when this work was completed.

[1] V. V. Flambaum, Phys. Scr. 46, 198 (1993); V.V. Flambaum and O.K. Vorov. Phys. Rev. Lett. 70, 4051 (1993); V.V. Flambaum in Time Reversal Invariance and Parity Violation in Neutron Reactions, edited by C.R. Gould, J.D. Bowman and Yu.P. Popov (World Scientific, Singapore, 1994), p. 39.

[2] V. V. Flambaum, A. A. Gribakina, G. F. Gribakin, and M. G. Kozlov, Phys. Rev. A 50, 267 (1994).

[3] V.V.Flambaum, G.F.Gribakin and F.M.Izrailev, Phys. Rev. E 53, 5729 (1996).

[4] V.V.Flambaum and F.M.Izrailev, Phys. Rev. E 55, R13 (1997); E 56, 5144 (1997).

[5] M.Horoi, V.Zelevinsky and B.A.Brown, Phys. Rev. Lett. 74, 5194 (1995); V.Zelevinsky, M.Horoi and B.A.Brown, Phys. Lett. B 350, 141 (1995); N.Frazier, B.A.Brown and V.Zelevinsky, Phys. Rev. C 54, 1665 (1996); V.Zelevinsky, B.A.Brown, M. Horoi and N.Frazier, Phys. Rep., 276 , 85 (1996).

[6] V.V.Flambaum, F.M.Izrailev, and G.Casati, Phys. Rev. E 54, 2136 (1996).

[7] A. Bohr and B. Mottelson, Nuclear structure, Vol. 1 (Benjamin, New York, 1969).

[8] B. Lauritzen, P.F. Bortignon, R.A. Broglia, and V.Zelevinsky, Phys. Rev. Lett. 74, 5190 (1995).

[9] B.W. Bush, G.F. Bertch and B.A. Brown. Phys. Rev. C 45, 1709 (1992).

[10] B.L.Altshuler, Y.Gefen, A.Kamenev and L.S.Levitov, Phys. Rev. Lett., 78, 2803 (1997); D.L.Shepelyansky and O.P.Sushkov, Europhys. Lett. 37, 121 (1997); A.D.Mirlin and Y.V.Fyodorov, Phys. Rev. B 56, 13393 (1997); V.V.Flambaum, G.F.Gribakin and O.P.Sushkov, 1997, unpublished; P.Jacquod and D.L.Shepelyansky, Phys. Rev. Lett. 79, 1837 (1997); V.V.Flambaum and G.F.Gribakin, Phys. Rev. C 50, 3122 (1994).

[11] J. B. French and S. S. M. Wong, Phys. Lett. B 35, 5 (1970). J.B. French, V.K.B. Kota, A. Pandey, and S. Tomsovic, Ann. Phys. (N.Y.) 181 (1988) 235. 